\title{
Determinação da temperatura Kondo a partir de curvas de susceptibilidade magnética em regime não líquido de Fermi
}

\author{
Determination of the Kondo temperature from the \\ curves of magnetic susceptibility on a non-Fermi \\ liquid regime
}

Lucas Carvalho Pereira, João Vítor Batista Ferreira.

\author{
Centro de Ciências Exatas e Tecnológicas - CCET - UFMS, Campo Grande, MS \\ e-mail: lukas.cp@gmail.com; jvitor2@dfi.ufms.br
}

\section{RESUMO}

Em algumas ligas metálicas existem átomos magnéticos cercados por vários outros não magnéticos. Os primeiros são tão poucos que não interagem entre si. Os segundos são maioria e formam uma banda de condução. Nestas ligas é comum a ocorrência de um fenômeno conhecido como Efeito Kondo. Trata-se do acoplamento entre o spin do átomo magnético com os spins dos elétrons da banda de condução. Este fenômeno, em baixas temperaturas, é perceptível em propriedades como suscetibilidade magnética, calor específico eletrônico, resistividade elétrica, entre outros; e é caracterizado por um parâmetro denominado Temperatura Kondo. $\mathrm{O}$ valor deste parâmetro indica a faixa de temperatura em que o Efeito Kondo passa a ser significativo. Sua determinação é importante para a compreensão de compostos metálicos em baixa temperatura, como $\mathrm{Ce}_{0.5} \mathrm{La}_{0.5} \mathrm{Ni}_{9} \mathrm{Ge}_{4}, U R u_{2} \mathrm{Si}_{2}$, entre outros. Krishna-murthy e colaboradores, através de cálculos perturbativos do modelo de Anderson para ligas magnéticas diluídas, propuseram uma tabela de dados de suscetibilidade magnética a partir da qual é possível a determinação da temperatura Kondo para sistemas físicos no regime líquido de Fermi. Neste artigo foi reproduzido este procedimento utilizando regressão numérica para ajustar um tipo de equação não-linearizável aos dados desta tabela. Em seguida, determinou-se a temperatura Kondo de um modelo de Anderson de dois canais no regime não líquido de Fermi. Confirmou-se que o método numérico proposto por Krishna-murthy e colaboradores é válido para os dois regimes.

Palavras-chave: Efeito Kondo, temperatura Kondo, susceptibilidade magnética, regressão numérica.

\section{ABSTRACT}

Metallic alloys can have magnetic atoms surrounded by several others non-magnetic. The first are so few that there is no interaction between them. The second are predominant and they form a metallic conduction band. In these alloys the occurrence of a phenomenon known as Kondo effect is common. It is the coupling of the spin of the magnetic atom and the spins of electrons in the conduction band. This phenomenon, at low temperatures, is perceptible in properties such as magnetic susceptibility, electronic specific heat, electrical resistivity, etc., and it is characterized by a parameter denominated Kondo temperature. The value of this parameter indicates the temperature range in which the Kondo effect becomes significant. Its determination is important for the understanding of metallic compounds in low temperature, such as $\mathrm{Ce}_{0.5} \mathrm{La}_{0.5} \mathrm{Ni}_{9} \mathrm{Ge}_{4}, U R u_{2} \mathrm{Si}_{2}$, etc. Krishna-murthy et al, through perturbative calculations of Anderson model for dilute magnetic alloys, proposed a table of magnetic susceptibility data from which it is possible to determine the Kondo temperature for physical systems in the Fermi liquid regime. In this article we reproduced this procedure by using numerical regression to adjust a type of non-linear equation to the data of this table. Next, we determined the Kondo Temperature of a two-channel Anderson model in non-Fermi liquid regime. We confirmed that the numerical method proposed by Krishna-murthy et al is valid for both regimes.

Keywords: Kondo effect, Kondo temperature, magnetic susceptibility, numerical regression. 


\section{INTRODUÇÃO}

\subsection{Impurezas magnéticas em metais - Efeito Kondo}

O estudo para desenvolvimento de dispositivos eletrônicos aproveitando os spins e as propriedades magnéticas dos elétrons (spintrônica) é um ramo de pesquisa importante atualmente. A compreensão do Efeito Kondo, fenômeno pesquisado desde as primeiras décadas do século XX, é essencial para este desenvolvimento [1].

A partir de 1930, com o avanço das técnicas de resfriamento, começou-se a constatar experimentalmente um comportamento anômalo na resistividade elétrica em algumas amostras metálicas com elevado grau de pureza [2]. Já era observado nos metais o decaimento monotônico da resistividade à medida que a temperatura $(T)$ tendia a zero. Isto era explicado pela Mecânica Quântica como diminuição da vibração da rede cristalina. Porém, em algumas amostras, observava-se o decaimento até uma determinada temperatura $(\sim 10 K)$ e, em seguida, um aumento, causando um mínimo na resistividade elétrica (Figura 1).

Verificou-se experimentalmente que esse mínimo na curva de resistividade elétrica estava relacionado com a presença de impurezas magnéticas no metal hospedeiro. Em geral, essas impurezas eram átomos isolados na rede cristalina do material e pertenciam aos elementos de transição ou terras-raras da tabela periódica (por exemplo, átomos de ferro em uma amostra de ouro). A concentração destas impurezas era tão baixa que se concluiu que importava apenas a interação delas com os elétrons de condução e não entre as mesmas.

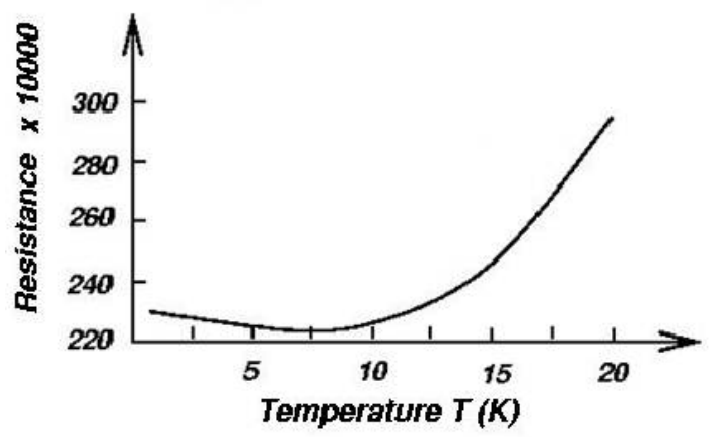

Figura 1: Representação da curva de resistividade elétrica do Ouro com mínimo antes da temperatura zero, obtido a partir de W. J. de Haas and G. J. van den Berg, Physica vol. 3, page 440, 1936.

Em 1964, J. Kondo obteve sucesso na explicação desse fenômeno ao propor um modelo de impureza magnética com apenas um elétron no orbital de valência, imerso em uma banda de condução não magnética [3] ]. $\mathrm{O}$ spin deste elétron pode mudar sua orientação devido ao espalhamento (colisões) dos elétrons de condução na impureza magnética. Este modelo é apresentado na Fig. 2.

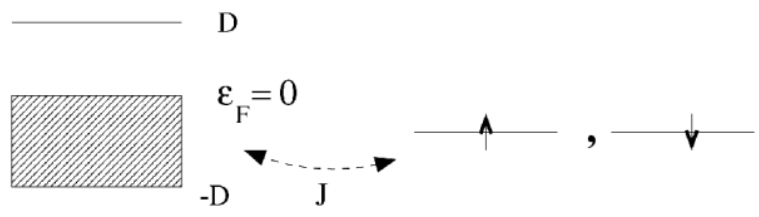

Figura 2: Modelo de Kondo. A banda de condução é semipreenchida, isotrópica e de largura $2 D(D \sim 5 e V)$, e atribuímos energia zero para o nível de Fermi $\left(\varepsilon_{F}=0\right)$. A impureza magnética é representada apenas pelo seu spin resultante $S=1 / 2$, com componentes $S_{z}=+1 / 2$ ou $S_{z}=-1 / 2$. O espalhamento dos elétrons de condução provoca a mudança entre estes dois estados quânticos $(|1 / 2,+1 / 2\rangle \leftrightarrow|1 / 2,-1 / 2\rangle)$. $J$ é um parâmetro que representa o acoplamento antiferromagnético entre o spin da impureza e os spins dos elétrons de condução.

Esta maneira de representar um momento magnético localizado já existia e era conhecida como modelo $s-d$ isotrópico, que mais tarde passou a ser conhecido como Modelo de Kondo. A principal característica é a valência fixa: a impureza nunca perde o elétron, que tem $\operatorname{spin} S=1 / 2$ e componentes $S_{z}=$ 
$+1 / 2$ ou $S_{z}=-1 / 2$. Através do acoplamento antiferromagnético entre o spin da impureza e o spin dos elétrons de condução, a impureza magnética pode mudar de estado quântico $(|1 / 2,+1 / 2\rangle \leftrightarrow|1 / 2,-1 / 2\rangle)$. A intensidade deste acoplamento é dada pelo parâmetro $J$ : é ele que faz com que o componente $z$ do spin do elétron da impureza "flipe" constantemente entre os dois estados. À medida que a temperatura diminui, este acoplamento aumenta a resistência elétrica do material.

Quando a temperatura é suficientemente baixa, a agitação térmica dos elétrons de condução é vencida pelo acoplamento antiferromagnético entre estes e a impureza: o spin da impureza magnética é completamente blindado pelo spin resultante dos elétrons de condução em sua volta. Este conjunto se comporta como uma partícula sem spin e não magnética.

Baseado nestas ideias, J. Kondo diagonalizou o Hamiltoniano deste modelo através de cálculos perturbativos e deduziu a existência de um mínimo na expressão da resistividade elétrica, justificando assim o resultado experimental. Apesar desse sucesso, a solução perturbativa de Kondo resultou no aparecimento de um termo logarítmico na equação da resistividade elétrica. Este termo diverge a partir de um dado valor de temperatura e este valor que ficou conhecido como Temperatura Kondo $\left(T_{K}\right)$. Este parâmetro é característico de cada sistema físico e indica a faixa de temperatura em que o acoplamento entre a impureza e os estados de condução passa a ser significativo. Este comportamento divergente da equação da resistividade não era constatado experimentalmente e concluiu-se tratar de um erro originário do método perturbativo, ineficiente para resolver o Hamiltoniano de Kondo em temperaturas muito baixas. Medidas de outras grandezas físicas mostraram que também havia anomalias devido à interação entre a impureza magnética e os elétrons de condução, como, por exemplo, calor específico eletrônico e suscetibilidade magnética.

No Modelo de Kondo, a Temperatura Kondo está relacionada com outras grandezas do modelo pela expressão.

$$
k_{B} T_{K} \sim D e^{-\frac{1}{2} \mathrm{~J} \rho_{0}},
$$

sendo $D$ a semilargura da banda de condução, $k_{B}$ a constante de Boltzmann, $\rho_{0}$ a densidade de estados eletrônicos próximo ao nível de Fermi e $J$ o parâmetro de hibridização entre a impureza e os elétrons de condução [2]. Esta expressão não é útil para se determinar o valor da Temperatura Kondo, pois $J$ e $\rho_{0}$ são difíceis de serem determinados experimentalmente.

Em 1961, portanto poucos antes de Kondo propor seu modelo, P. W. Anderson havia proposto outro modelo para descrever ligas magnéticas diluídas, sem vinculá-lo explicitamente ao comportamento anômalo da resistividade elétrica [4]. Ao contrário do Modelo de Kondo, em que a valência da impureza é fixa, no Modelo de Anderson a impureza pode ser ionizada (Figura 3).

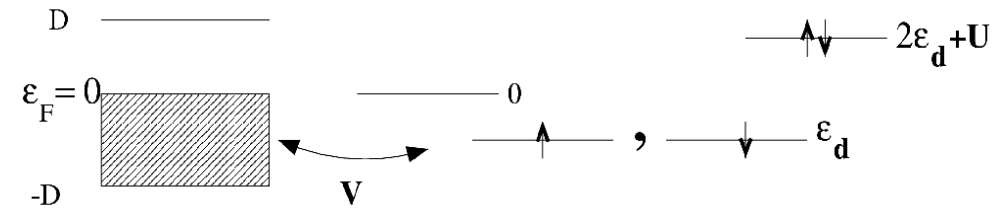

Figura 3: Modelo de Anderson. A banda de condução do metal é idêntica ao do Modelo de Kondo. A impureza é representada pelas suas quatro configurações de valência possíveis, sendo $\varepsilon_{\boldsymbol{d}}$ a energia do orbital quando ocupado com apenas um elétron e $2 \varepsilon_{\boldsymbol{d}}+\boldsymbol{U}$ quando ocupado por dois elétrons. $\boldsymbol{V}$ é um parâmetro que representa a energia de hibridização entre a impureza e a banda de condução (equivale ao parâmetro $\boldsymbol{J}$ no Modelo de Kondo). $\boldsymbol{U}$ é a repulsão Coulombiana entre os dois elétrons no mesmo nível. Se $\mathbf{2} \varepsilon_{\boldsymbol{d}}+\boldsymbol{U}=\mathbf{0}$, temos o chamado Modelo de Anderson simétrico, que exibe simetria partícula-buraco (estado duplamente ocupado tem a mesma energia do estado vazio).

No Modelo de Anderson a impureza magnética pode ter seu orbital de valência vazio, com um ou dois elétrons, sendo que a banda de condução recebe ou fornece esses elétrons. Em seu estado fundamental, a impureza tem apenas um elétron cujo spin é $S=1 / 2$ e componentes $S_{z}=+1 / 2$ ou $S_{z}=-1 / 2$. No Modelo de Anderson a impureza pode perder um elétron com $S_{z}=+1 / 2$ e depois ganhar um elétron com $S_{z}=-1 / 2$, sendo a intensidade deste processo indicada pelo parâmetro de hibridização $V$. O resultado final é equivalente à alternância de estados $(|1 / 2,+1 / 2\rangle \leftrightarrow|1 / 2,-1 / 2\rangle)$ do Modelo de Kondo. Além disso, no Modelo de Anderson existe a possibilidade da impureza não perder e ainda assim ganhar mais um elétron. Neste caso, a energia do estado quântico duplamente ocupado leva em consideração a repulsão Coulombiana entre os dois 
elétrons, indicada por $U$. Arbitrando-se que a energia $2 \varepsilon_{d}+U$ é igual a zero, este modelo fica conhecido como Modelo de Anderson simétrico.

J. Schrieffer e P. Wolff [ $\underline{5}$ ] demonstraram matematicamente que o Modelo de Kondo era um caso particular do Modelo de Anderson. Em 1975, o Hamiltoniano do Modelo de Kondo foi solucionado numericamente sem aproximações pela técnica conhecida como Grupo de Renormalização Numérica [6]. O resultado numérico não apresentou a divergência logarítmica e coincidiu com os dados experimentais. Cinco anos depois, também usando a mesma técnica, Krishna-murthy e colaboradores diagonalizaram o Hamiltoniano do Modelo de Anderson [7]. A solução analítica do Modelo de Kondo foi obtida independentemente por N. Andrei (1980) e P. B. Wiegmann (1981) utilizando ansatz de Bethe [ $\underline{8}, \underline{9}]$.

P. W. Anderson observou que em seu modelo também ocorre, em temperatura suficientemente baixa $\left(T \ll T_{K}\right)$, a blindagem do spin da impureza magnética pelos spins dos elétrons de condução e a perda do caráter magnético da impureza (Figura 4). Este fenômeno é característico de todos os sistemas que contêm átomos magnéticos circundados por átomos que formam uma banda de condução e passou a ser conhecido como Efeito Kondo.

Também importante foi a constatação, a partir de 1950, que, em temperatura inferior àquela em que ocorre o Efeito Kondo $\left(T \ll T_{K}, T \rightarrow 0\right)$, os metais simples apresentam propriedades físicas cujo comportamento é conhecido como regime líquido de Fermi. O efeito Kondo influencia o comportamento de líquido de Fermi dos elétrons de condução. A Teoria do Líquido de Fermi é uma aproximação que assume que, em baixas energias, os elétrons de condução de um metal se comportam como um conjunto de partículas fracamente interagentes, parecido com um gás de elétrons. L. Landau introduziu esta ideia em 1956 baseando-se no estudo que E. Fermi realizou com o líquido neutro do isótopo $\mathrm{He}^{3}$. Para os elétrons nos metais foi dado um tratamento apropriado devido aos efeitos da interação Coloumbiana. Todas as evidências apontavam para o fato desta ser uma teoria direta e preferencialmente aplicável aos metais simples, como os alcalinos [10]. Segundo Landau, o estudo dos metais pode se utilizar do conceito de gás de elétrons, e este conceito é notadamente útil para explicar as propriedades cinéticas dos elétrons de condução. Essa descrição dos metais é muitas vezes denominada de "gás de elétrons de Fermi", um gás de elétrons sujeito ao Princípio de Exclusão de Pauli. O dilema fundamental é: como se pode usar um modelo de elétrons livres se as interações eletrônicas são muito fortes? É neste ponto que entra a sutil aproximação da teoria do líquido de Fermi feita por Landau. Inicialmente se reconhece que, de fato, o modelo de elétrons livres não é uma boa aproximação. No entanto, em vez de elétrons, o enfoque é dado em novas entidades denominadas de quasipartículas. Neste contexto, a quasipartícula é formada por um elétron e pela nuvem eletrônica ao seu redor, responsável pela blindagem do seu campo coulombiano pelos outros elétrons. Assim, o modelo de quasipartículas livres é válido, pelo menos para níveis de energia próximos do nível de Fermi. Isso é suficiente para minimizar os efeitos da interação coulombiana elétron-elétron. A maioria dos metais simples apresenta comportamento previsto por esses conceitos e por isso é dito que eles se comportam de acordo com o regime líquido de Fermi (LF). A partir da década de 1990, novas ligas metálicas complexas começaram a ser estudadas e produzidas em laboratório, e descobriu-se que apresentam comportamento diferente daquele previsto pelo regime LF. Passou-se então a denominar de regime não líquido de Fermi (nLF) toda variedade de comportamentos que diferem da teoria de Landau [11].

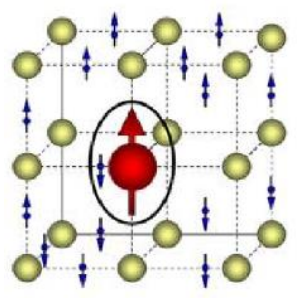

Figura 4: Blindagem Kondo. O spin 1/2 da impureza é completamente blindado pelo spin resultante dos elétrons da banda de condução em temperatura suficientemente baixa $\left(T \ll T_{K}\right)$.

Tanto o modelo de impureza magnética proposto por Kondo, como o modelo proposto por Anderson apresentam propriedades físicas com comportamento de acordo com o regime LF. Ao solucionar o Modelo de Anderson pela técnica do Grupo de Renormalização Numérica, Krishna-murthy e colaboradores desenvolveram um procedimento numérico para se determinar a Temperatura Kondo a partir da curva de suscetibilidade magnética em função da temperatura no regime LF. Esta propriedade física indica o quão suscetível é 
um material de sofrer influência de um campo magnético externo. Em termos da Mecânica Quântica, a suscetibilidade magnética $(\chi)$ de um sistema atômico é definida pela relação

$$
\frac{k_{B} T \chi}{\left(g \mu_{b}\right)^{2}}=\left\langle S_{Z}^{2}\right\rangle-\left\langle S_{Z}\right\rangle^{2},
$$

na qual $\left\langle S_{z}\right\rangle$ é o valor esperado do componente $z$ do spin da impureza magnética, $k_{B}$ é a constante de Boltzmann, $\mu_{B}$ é o magnéton de Bohr e $g$ é o fator giromagnético do elétron. Krishna-murthy e colaboradores descobriram que curvas de

$$
\frac{k_{B} T \chi}{\left(g \mu_{B}\right)^{2}} \text { versus } \ln \left(\frac{T}{T_{K}}\right) \text {, }
$$

são idênticas em baixa temperatura, isto é, exibem um comportamento universal. Como já mencionado, o parâmetro $T_{K}$ é característico de cada sistema físico, ou seja, depende do valor de $J$ nos Modelos de Kondo (e de outros parâmetros). Através de expansões perturbativas, eles propuseram uma tabela de dados e um procedimento para determinar graficamente o valor aproximado de $T_{K}$.

Atualmente, outros modelos procuram descrever compostos metálicos que apresentam comportamento exótico, isto é, comportamento não líquido de Fermi (nLF). Neste artigo utilizou-se o Modelo de Anderson de Dois Canais (MADC), proposto por D. Cox (veja Figura 5 na seção 4). Este modelo apresenta facilmente regimes LF e nLF através de variação de alguns parâmetros.

Este artigo corrobora o resultado obtido na Ref. [20], de que o procedimento prescrito por Krishnamurthy para determinar os valores da Temperatura Kondo no regime LF também funciona no regime nLF; e usa este procedimento e regressão numérica nos dados de tabela fornecida por Krishna-murthy e colaboradores.

\subsection{Regressão Numérica}

A regressão numérica é uma ferramenta essencial para se extrair a dependência entre grandezas físicas através de dados experimentais ou numéricos. Na literatura, essa técnica é bem conhecida para o caso em que esta dependência é linear (equação da reta) ou logarítmica (equação da exponencial) [12]. Mas existem situações em que a dependência não é trivial, sendo a equação-proposta não passível de ser reduzida ao caso linear [13]. Hoje em dia o ajuste de curvas ao conjunto de pontos experimentais (regressão numérica ou "fitting") é uma ferramenta onipresente nos pacotes computacionais gráficos ou matemáticos. Mais recente é a opção de "computação nas nuvens", em que se pode acessar um site e utilizar-se das mais modernas ferramentas disponíveis para esta técnica [14]. Em áreas como Estatística, Química e principalmente Biologia, estas opções são mais que suficientes, pois uma grande parte dos fenômenos estudados pode ser descritos pela equação da reta ou equação da exponencial simples, que são de fácil compreensão e interpretação. Mas em física é comum aparecerem situações em que as grandezas físicas envolvidas não estão relacionadas de maneira diretamente proporcional ou exponencial simples. Nestes casos é importante lembrar que os pacotes computacionais têm um conjunto de equações disponíveis para o ajuste que pode não satisfazer aos dados a serem analisados.

Neste artigo utiliza-se uma técnica de regressão numérica baseada em procedimento recursivo que mantém a simplicidade da dependência linear, mas que é adaptável para equações não linearizáveis como, por exemplo, equações transcendentais. Esse procedimento foi aplicado para determinar os parâmetros $a$ e $b$ da expressão

$$
y=\frac{a x^{b}}{1+x}
$$

de forma a ajustá-la ao conjunto de pontos numéricos dados por Krishna-murthy e colaboradores na determinação da Temperatura Kondo no Modelo de Anderson. Este artigo mostra como o uso do procedimento recursivo pode ser útil na elaboração de códigos computacionais simples, que calculam os parâmetros que minimizam o erro para equações não linearizáveis.

Na seção 2 encontra-se uma revisão dos conceitos fundamentais de regressão numérica pelo método dos mínimos quadrados. Na seção 3 tem-se a regressão dos dados numéricos de Krishna-murthy e colaboradores através da Eq. 4 e, a partir daí, determinou-se o valor da expressão $\left(T_{K}\right.$ - temperatura Kondo): 


$$
\frac{k_{B} T_{K} \chi}{\left(g \mu_{B}\right)^{2}}
$$

Na seção 4 apresenta-se o Modelo de Anderson de Dois Canais. Com as curvas de suscetibilidade magnética em regime nLF deste modelo, encontrou-se os respectivos valores de Temperatura Kondo, e verifica-se que estas curvas apresentam comportamento universal em baixa temperatura. Na seção 5 as conclusões são apresentadas.

\section{REGRESSÃO NUMÉRICA: MÉTODO DOS MÍNIMOS QUADRADOS}

O Método dos Mínimos Quadrados (MMQ) [12] é usado para o ajuste da curva que representa uma equação matemática com uma tabela de pontos experimentais ou numéricos $\left(x_{i}, y_{i}\right)$, sendo que o principal objetivo é obter os valores dos parâmetros desta equação que melhor ajusta a este conjunto de pontos. É importante enfatizar que a equação de ajuste é uma escolha arbitrária; o método apenas descobre os melhores parâmetros desta equação para o ajuste.

O caso mais simples do MMQ é o ajuste linear. Os pontos podem ser representados graficamente por uma reta no plano cartesiano. Por isso, a equação proposta é

$$
y=a x+b,
$$

sendo $a$ e $b$ os parâmetros a serem determinados. Por exemplo, sejam $n$ pontos experimentais ou numéricos que são representados por $\left(x_{i}, y_{i}\right)$. Os pontos que serão obtidos teoricamente pela candidata a melhor expressão é representada por $\left(x_{i}, y\right)$. Neste caso, o interesse está em minimizar a "distância" entre $y_{i}$ e $y$.

$$
\left|y_{i}-y\right|=\left|y_{i}-\left(a x_{i}+b\right)\right| \text {. }
$$

Ao valor da soma dos quadrados dessas distâncias define-se como erro quadrático:

$$
q=\sum_{i=1}^{n}\left(y_{i}-a x_{i}-b\right)^{2} .
$$

Na tentativa de minimizar a Eq. 8, a mesma foi derivada parcialmente em relação aos parâmetros $a$ e $b$ e igualada a zero.

$$
\begin{aligned}
& \frac{\partial q}{\partial a}=\sum_{i=1}^{n} 2\left(y_{i}-a x_{i}-b\right)\left(-x_{i}\right)=0 \mathrm{e} \\
& \frac{\partial q}{\partial b}=\sum_{i=1}^{n} 2\left(y_{i}-a x_{i}-b\right)(-1)=0 .
\end{aligned}
$$

Após pequena álgebra, obtém-se um sistema de duas equações e duas incógnitas,

$$
\left\{\begin{array}{c}
\left(\sum_{i=1}^{n} y_{i} x_{i}\right)=\left(\sum_{i=1}^{n} x_{i}^{2}\right) a+\left(\sum_{\mathrm{i}=1}^{\mathrm{n}} x_{i}\right) b \\
\left(\sum_{i=1}^{n} y_{i}\right)=\left(\sum_{i=1}^{n} x_{i}\right) a+n b
\end{array}\right.
$$

no qual, após sua resolução, encontramos os valores dos parâmetros $a$ e $b$, que minimizam o erro quadrático. Observe que o procedimento consiste apenas em resolver um sistema de duas equações e duas incógnitas: todos os valores de $\left(x_{i}, y_{i}\right)$ são conhecidos e resta encontrar os valores de $a$ e $b$. A equação da reta é a expressão matemática mais simples e intuitiva para se minimizar o erro quadrático.

Esse método é muito usado na literatura científica para modelos com comportamento linear. Também é viável aplicar o MMQ em expressões do tipo

$$
\begin{aligned}
& y=a e^{b x}, \\
& y=\frac{1}{a+b x}, \\
& y=a x^{b} \mathrm{e} \\
& y(x)=a_{0}+a_{1} x+a_{2} x^{2}+\cdots+a_{n} x^{n},
\end{aligned}
$$


que são denominadas de equações linearizáveis. Nestas equações, fazendo algumas mudanças de variáveis como, por exemplo, a logaritmização de um exponencial, retorna-se ao caso de ajuste de uma equação da reta. Apresenta-se a seguir um método para regressão de equação não-linear.

\section{REGRESSÃO NUMÉRICA PARA EQUAÇÕES NÃO-LINEARES: \\ DEPENDÊNCIA DE $k_{B} T \chi /\left(g \mu_{B}\right)^{2}$ versus $T / T_{K}$}

Segundo Krishna-murthy e colaboradores, quando $T=T_{K}$, a Eq. 5 é igual a 0,0701(Tabela V e Figura 11 da Ref. [7].

Observa-se que existe um erro de grafia na legenda da Figura 11 desta referência: em vez de 0,701 leia-se 0,0701 .

Tabela 5: Tabela V retirada do artigo de Krishna-murthy e colaboradores [7]: estes são os dados para a regressão numérica.

\begin{tabular}{lcc}
\hline \hline & & \\
$T / T_{K}$ & $\log _{10}\left(T / T_{K}\right)$ & $\frac{k_{B} T \chi}{\left(g \mu_{\mathrm{B}}\right)^{2}}$ \\
& & \\
\hline & & \\
28.270 & 1.451 & 0.173 \\
18.851 & 1.275 & 0.166 \\
12.565 & 1.099 & 0.157 \\
8.379 & 0.923 & 0.147 \\
5.585 & 0.7470 & 0.135 \\
3.723 & 0.5709 & 0.122 \\
2.482 & 0.3958 & 0.107 \\
1.655 & 0.2187 & 0.0910 \\
1.103 & 0.0426 & 0.0739 \\
0.735 & -0.1335 & 0.0580 \\
0.490 & -0.3098 & 0.0430 \\
0.327 & -0.4859 & 0.0310 \\
0.218 & -0.6620 & 0.0213 \\
0.145 & -0.8388 & 0.0145 \\
0.0969 & -1.014 & 0.0095 \\
& & \\
\hline \hline
\end{tabular}

Tabela 2: Dados retirados da Tabela V da Ref. []] de Krishna-murthy e colaboradores. Foi feita regressão não-linear para determinar o valor de $k_{B} T \chi /\left(g \mu_{B}\right)^{2}$ quando $T / T_{K}=1,0$.

\begin{tabular}{l|l}
\hline $\mathbf{T} / \mathbf{T}_{\mathbf{K}}$ & $\mathbf{k}_{\mathbf{B}} \mathbf{T}_{\mathbf{X}} /\left(\mathbf{g} \mu_{\mathbf{B}}\right)^{2}$ \\
\hline 28,270 & 0,173 \\
\hline 18,851 & 0,166 \\
\hline 12,565 & 0,157 \\
\hline 8,379 & 0,147 \\
\hline 5,585 & 0,135 \\
\hline 3,723 & 0,122 \\
\hline 2,482 & 0,107 \\
\hline 1,655 & 0,0910 \\
\hline 1,103 & 0,0739 \\
\hline 0,735 & 0,0580 \\
\hline 0,490 & 0,0430 \\
\hline 0,327 & 0,0310 \\
\hline 0,218 & 0,0213 \\
\hline 0,145 & 0,0145 \\
\hline 0,0969 & 0,0095 \\
\hline
\end{tabular}


Baseado na Ref. [7] foi proposta a equação não-linear $\left(k_{B} T \chi /\left(g \mu_{B}\right)^{2}=y, T / T_{K}=x\right)$ :

$$
y=\frac{a x^{b}}{1+x}
$$

em que $a$ e $b$ são parâmetros de ajuste, de modo que a curva desta equação possa se ajustar aos pontos da Tabela 1. A Eq. 7 é parecida com a expressão Curie-Weiss para a susceptibilidade magnética

$$
\chi=\frac{a}{b+T}
$$

diferindo pelo termo não linear da temperatura. A Eq. 16 não se enquadra em nenhuma das equações linearizáveis citadas na seção anterior, ou seja, não é possível aplicar diretamente o MMQ ou fazer uma mudança de variável apropriada. Ao contrário da equação da reta, agora não se obtém apenas um sistema de duas equações para solucionar.

Desenvolveram-se dois métodos recursivos para a determinação dos parâmetros $a$ e $b$ : método recursivo direto e método recursivo otimizado. Para cada um deles construiu-se um programa computacional em linguagem $\mathrm{C}++$ e determinaram-se os parâmetros $a$ e $b$ que minimizam o erro quadrático $(q)$.

i) Método recursivo direto na equação do erro quadrático. Inicia-se este processo fazendo uma varredura dos parâmetros $a$ e $b$ através de um laço (também chamado de loop) com bissecção do intervalo, e calculando o erro quadrático até atingir um valor muito próximo de zero ou valor mínimo. Apesar da eficácia, o método recursivo direto exige um tempo grande de processamento. Define-se a grade de variação dos parâmetros em intervalos discretos muito pequenos para melhor precisão. Variações grandes acarretam diminuição no tempo de processamento, porém o programa pode divergir (loop infinito) ou não alcançar resultados satisfatórios. Como este método não apresentou eficiência, utilizou-se a técnica descrita a seguir. drático

ii) Método recursivo otimizado na derivada da equação do erro quadrático. $\mathrm{Na}$ expressão do erro qua-

$$
q=\sum_{i=1}^{10}\left(y_{i}-\frac{a x_{i}^{b}}{1+x_{i}}\right)
$$

Encontra-se a expressão da derivada parcial com relação ao parâmetro $a$ ou $b$. Por exemplo, derivando em relação ao parâmetro $a$, obtém-se:

$$
\frac{\partial q}{\partial a}=\sum_{i=1}^{10} 2\left(y_{i}-\frac{a x_{i}^{b}}{1+x_{i}}\right)\left(\frac{-x_{i}^{b}}{1+x_{i}}\right)=0 \text {. }
$$

Ajustando a expressão acima, foi obtido o valor de $a$ :

$$
\begin{aligned}
& \sum_{i=1}^{10} y_{i}\left(\frac{x_{i}^{b}}{1+x_{i}}\right)-a \sum_{i=1}^{10}\left(\frac{x_{i}^{b}}{1+x_{i}}\right)^{2}=0 \mathrm{e} \\
& a=\sum_{i=1}^{10} y_{i}\left(\frac{x_{i}^{b}}{1+x_{i}}\right) / \sum_{i=1}^{10}\left(\frac{x_{i}^{b}}{1+x_{i}}\right)^{2} .
\end{aligned}
$$

Derivando a expressão do erro quadrático em relação ao parâmetro $b$, obtém-se

$$
\frac{\partial q}{\partial b}=\sum_{i=1}^{10} 2\left(y_{i}-\frac{a x_{i}^{b}}{1+x_{i}}\right)\left(-\frac{a x_{i}^{b}}{1+x_{i}} \ln \left(x_{i}\right)\right)=0,
$$

resultando em uma equação transcendental em que não é possível isolar $b$,

$$
-\sum_{i=1}^{10} y_{i} \frac{a x_{i}^{b}}{1+x_{i}} \ln \left(x_{i}\right)+\sum_{i=1}^{10}\left(\frac{a x_{i}^{b}}{1+x_{i}}\right)^{2} \ln \left(x_{i}\right)=0 .
$$

As Eq. 21 e 23 mostram que não é possível encontrar os parâmetros através de um simples sistema de 
duas equações. Mas através da Eq. 21 pode-se fazer o processo recursivo: atribuindo-se valor para $b$, encontra-se $a$, que minimiza o erro quadrático. Estabelecendo uma grade para variação de $b$, determinam-se os melhores parâmetros para minimizar $q$. Esse procedimento é mais eficiente: o tempo de processamento é menor por causa da dependência entre os parâmetros, dada pela Eq. 21.

Os valores obtidos pelo método ii foram: $a=0,139765$ e $b=1,07571$. Na Fig. 5 mostra-se a boa concordância com os pontos da Tabela 1 e. abaixo, a Eq. 16 com os parâmetros encontrados:

$$
y=\frac{0,139765 x^{1,07571}}{(1+x)}
$$

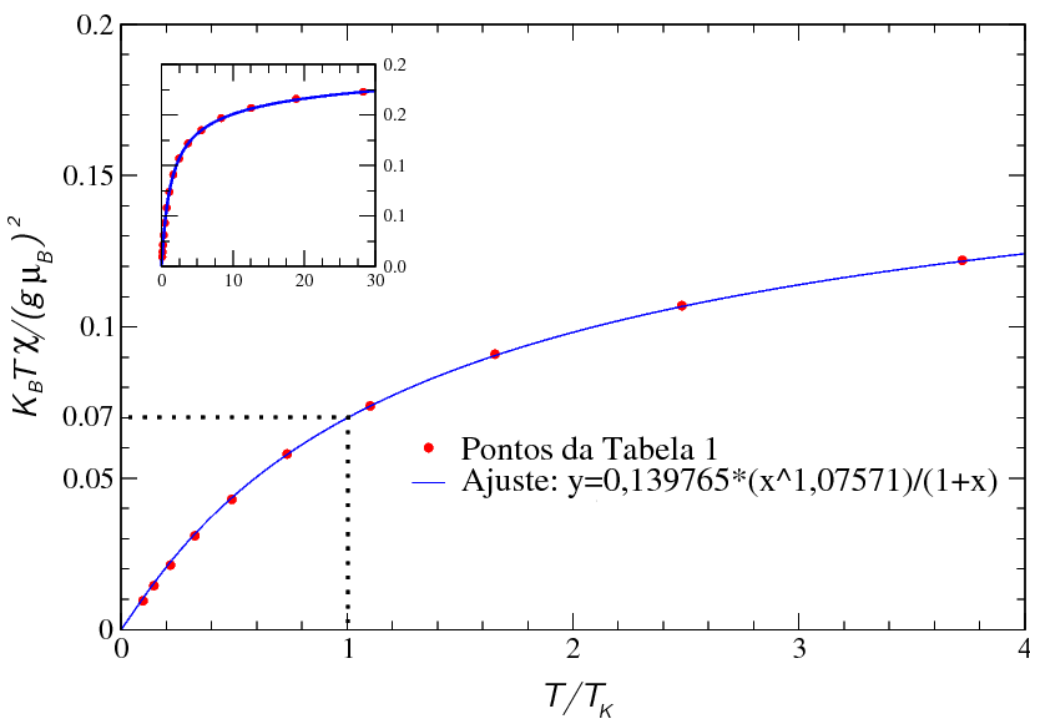

Figura 5: Os pontos representam os dados da Tabela 1 e a curva contínua, o ajuste obtido pela regressão numérica. Este e os gráficos seguintes foram desenhados pelo software livre Grace.

Usando a Eq. $24\left(y \equiv\left(k_{B} T \chi /\left(g \mu_{B}\right)^{2}, x \equiv T / T_{K}\right)\right.$ e fazendo $T / T_{K}=1,0$ obtém-se:

$$
\frac{k_{B} T_{K} \chi}{\left(g \mu_{B}\right)^{2}}=0,0698825
$$

Note agora que não se tem apenas os valores discretos da Tabela 1, mas uma "faixa contínua" entre os valores extremos (da própria Tabela 1) nos quais a Eq. 24 fornece com bastante precisão.

Este método recursivo otimizado para regressão numérica não linear será muito útil na determinação da dependência da Temperatura Kondo pelos parâmetros físicos do Modelo de Anderson de Dois Canais.

\section{MODELO DE ANDERSON DOIS CANAIS: CURVAS DE SUSCETIBILIDADE MAGNÉTICA E RESPECTIVOS VALORES DE TEMPERATURA KONDO NO REGIME NLF.}

Como visto na Introdução, o Efeito Kondo é resultado da interação de impureza magnética com os elétrons da banda de condução do metal. Dentre vários modelos de impurezas magnéticas, neste artigo foi utilizado o Modelo de Anderson de Dois Canais (MADC), sendo que o Modelo de Anderson tradicional (um canal, [4] ) é um caso particular [15].

No MADC o estado fundamental da impureza de Anderson tem $m$ elétrons e spin total $S=1 / 2(\sigma=$ $\left.S_{z}=+1 / 2,-1 / 2\right)$. Este estado pode perder ou receber um elétron para a banda de condução do metal. Seguindo a prescrição de Nozières e Blandim [16], considera-se que fatores como interação spin-órbita, campo cristalino, etc., resultam em duas ressonâncias na hibridização entre impureza e banda de condução. Cada ressonância é indicada por um número quântico denominado de canal e indicado por $\alpha=1,2(-\alpha=2,1)$. Os novos estados têm spin total zero e número quântico $\alpha$. Observe que se usa o termo "canal" para indicar esta ressonância em energia, e não como um sulco ou caminho no espaço físico do bulk do composto.

Na Figura 7 o metal é representado por uma banda de condução isotrópica, semipreenchida, de largura total $2 D(D \sim 5 e V)$. A impureza é representada por seus orbitais de valência, sendo o estado fundamental representado por um dubleto com $m$ elétrons, energia total $E_{0}$, canal $\alpha=0$ e $\operatorname{spin}$ total $\sigma=1 / 2$ ou $\sigma=-1 / 2$. 
Por simplicidade de notação, sempre que canal ou spin total são nulos, eles são omitidos. Nesse estado, a impureza pode ganhar ou perder um elétron para a banda através de ressonâncias de acordo com o canal. A intensidade deste processo de hibridização depende apenas do canal e é dada pelos parâmetros $V_{\alpha}\left(V_{1}\right.$ e $\left.V_{2}\right)$. Após a perda ou ganho de um elétron, a impureza fica em um estado excitado com energia total $E_{1}$, spin total nulo e canal $\alpha=1$ ou $\alpha=2$. São quatro estados excitados degenerados representados pelos dubletos $|m+1, \alpha\rangle e|m-1, \alpha\rangle$.

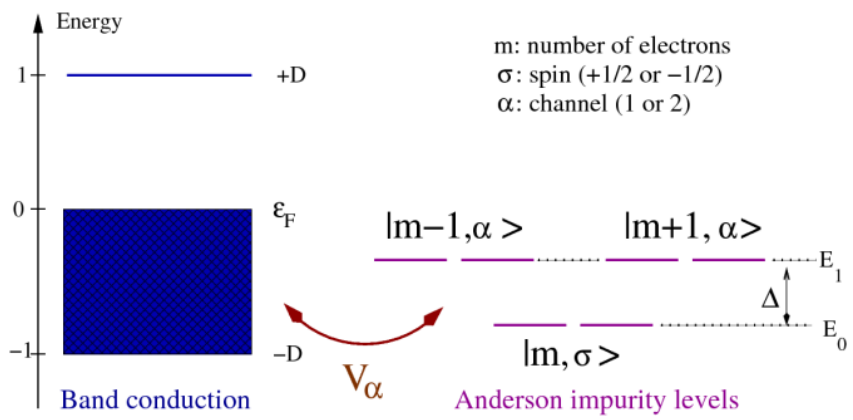

Figura 6: Modelo de Anderson de Dois Canais, figura retirada da ref. [19]: banda de condução e estados de valência da impureza. Do estado fundamental a impureza pode se hibridizar com a banda através de duas ressonâncias distintas (canais), ganhando ou perdendo um elétron. Cada canal tem intensidade determinada pelos parâmetros $V_{\alpha}\left(V_{1} e V_{2}\right)$. Os estados excitados têm energia $\Delta$ maior que o estado fundamental.

Os parâmetros $V_{1}$ e $V_{2}$ representam a intensidade de hibridização entre a impureza e a banda de condução, sendo que existe um valor para cada canal e a relação entre estes valores afeta o Efeito Kondo.

Quando $V_{1} \neq V_{2}$, o MADC representa um sistema em que o acoplamento indicado por um dos canais é mais intenso que o outro. $\mathrm{O}$ sistema neste regime caracteriza-se pelo fato do spin da impureza ser completamente blindado pelos spins dos elétrons de condução em baixa temperatura. Isto caracteriza o Efeito Kondo normal e o comportamento exibido em baixa temperatura é líquido de Fermi (LF). O Modelo de Anderson tradicional (um canal) sempre apresenta este regime.

Quando $V_{1}=V_{2}$ ocorre, o efeito Kondo é supercompensado, isto é, o spin da impureza não é completamente blindado pelos spins dos elétrons da banda de condução. O resultado é que o sistema apresenta comportamento não líquido de Fermi (nLF) em baixa temperatura [17]. As grandezas $\Delta=E_{1}-E_{0}, V_{1}$ e $V_{2}$ são os parâmetros físicos do MADC que, através do ajuste dos seus valores, permitem representar o sistema físico como LF ou nLF.

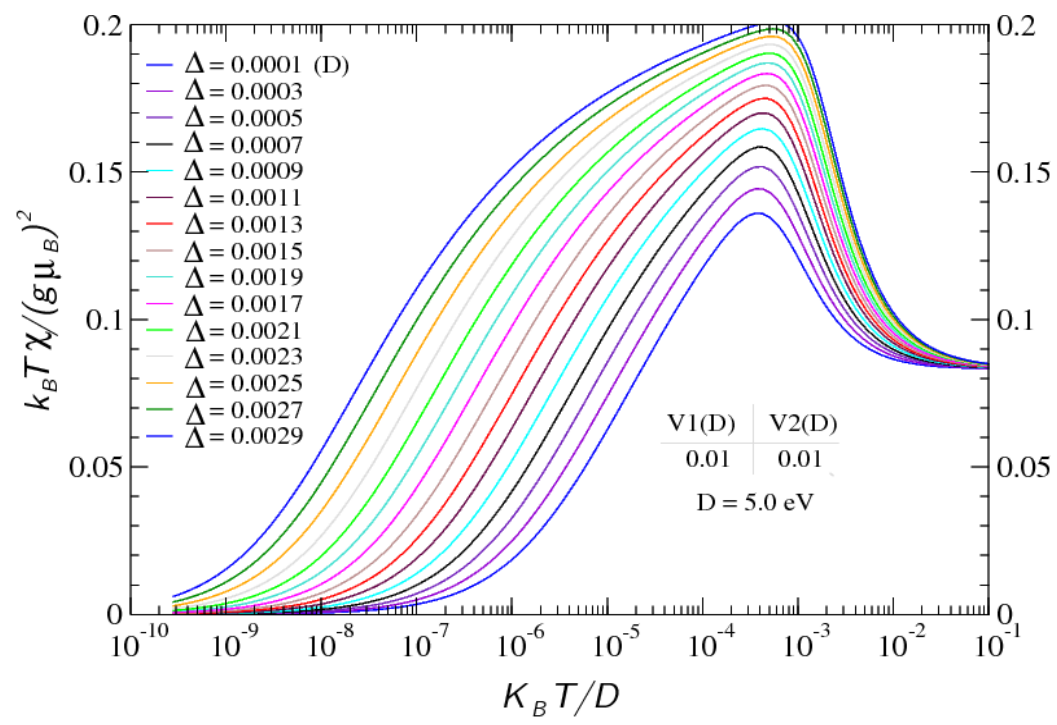

Figura 7: Curvas de suscetibilidade magnética do MADC para diferentes valores de $\Delta$ e todas no regime não líquido de Fermi (nLF) $V_{1}=V_{2}$. A partir destas curvas e usando a Eq. 25, determinou-se o valor da Temperatura Kondo para cada uma delas. 
O MADC utilizado neste trabalho foi diagonalizado usando Grupo de Renormalização Numérica conjuntamente com as técnicas associadas Intercalamento e Multi-Passos [18, $\underline{19}]$. Com os autovalores de energia e com os autoestados do sistema, calculam-se diversas propriedades físicas, como calor específico eletrônico e a suscetibilidade magnética em função da temperatura (Figura 8).

Determina-se a suscetibilidade magnética $\chi$ através da taxa de variação da magnetização $M$ pelo campo magnético externo $H$ :

$$
\chi=+\frac{\partial M}{\partial H}
$$

A magnetização pode ser calculada pela taxa de variação da Energia Livre de Helmholtz $F$ pelo campo magnético externo:

$$
M=-\frac{\partial F}{\partial H},
$$

Sendo

$$
F=-k_{B} T \ln (Z)
$$

e $Z$ a função de partição. Obtém-se a função de partição a partir dos autovalores de energia. Consequentemente,

$$
\chi=-\frac{\partial^{2} F}{\partial H^{2}} .
$$

O resultado final é a Eq. 2. Observa-se que a susceptibilidade magnética do MADC está intimamente ligada ao spin resultante do estado fundamental da impureza de Anderson hibridizada com os estados de condução.

Utilizando o procedimento descrito na seção anterior, determinou-se o valor da Temperatura Kondo para cada curva da Fig. 8, sendo o resultado mostrado na Tabela 2. Com os valores de Temperatura Kondo verificou-se que todas as curvas de suscetibilidade magnética apresentam o mesmo comportamento universal em baixa temperatura, bastando esboçar as mesmas curvas em função de $T / T_{K}$ (Figura 9) [20].

Tabela 1: Regime não líquido de Fermi (nLF) do MADC $\left(V_{1}=V_{2}=0,01 D, D=5 \mathrm{eV}\right)$. Cada valor de $\Delta$ representa uma curva diferente na Fig. 8, e foi determinado o respectivo valor de $T_{K}$.

\begin{tabular}{l|l}
\hline$\Delta(\mathrm{D})$ & $\mathrm{T}_{\mathrm{K}}(\mathrm{K})$ \\
\hline 0,0001 & 0,783 \\
\hline 0,0003 & 0,495 \\
\hline 0,0005 & 0,311 \\
\hline 0,0007 & 0,196 \\
\hline 0,0009 & 0,122 \\
\hline 0,0011 & 0,077 \\
\hline 0,0013 & 0,048 \\
\hline 0,0015 & 0,030 \\
\hline 0,0017 & 0,018 \\
\hline 0,0019 & 0,011 \\
\hline 0,0021 & 0,007 \\
\hline 0,0023 & 0,004 \\
\hline 0,0025 & 0,003 \\
\hline 0,145 & 0,0145 \\
\hline 0,0969 & 0,0095 \\
\hline
\end{tabular}


Observa-se que na Fig. 9 o comportamento universal no regime não líquido de Fermi é diferente do comportamento universal no regime líquido de Fermi.

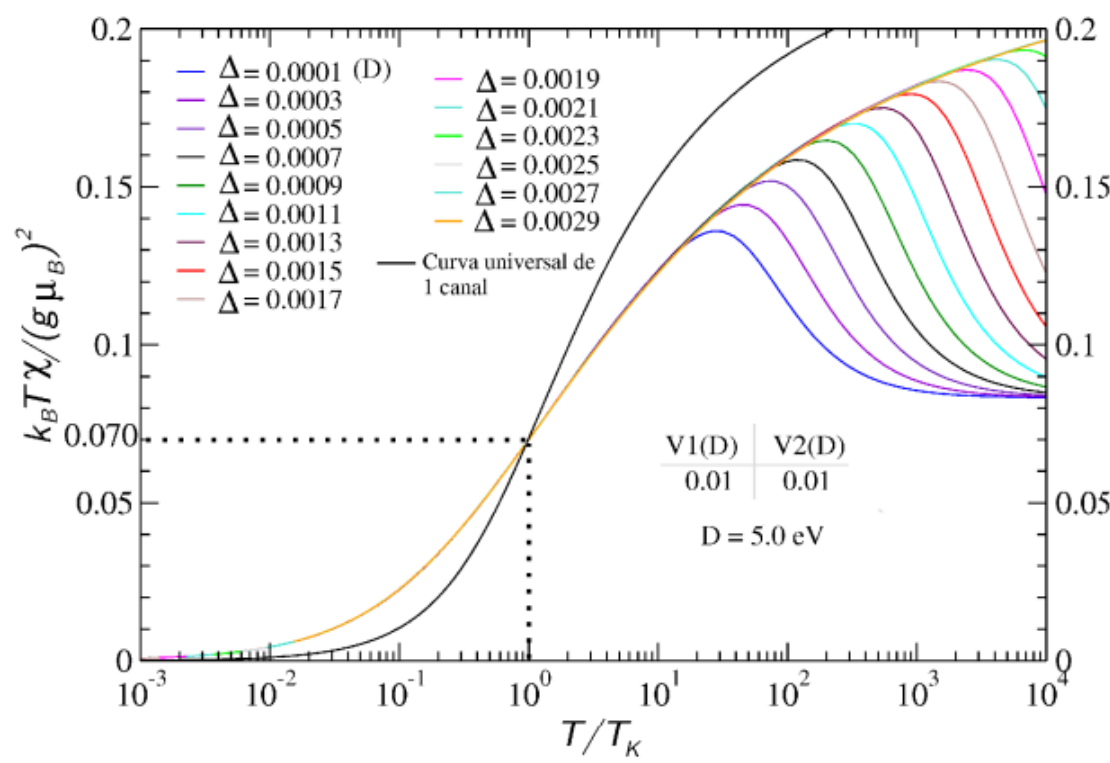

Figura 8: Comportamento universal em baixa temperatura da susceptibilidade magnética em função de $T / T_{K}$ do MADC no regime não líquido de Fermi (nLF). $O$ valor de $T_{K}$ foi determinado pelo procedimento numérico proposto por Krishnamurthy e colaboradores para modelos de Anderson no regime líquido de Fermi (LF), indicado pela curva universal do Modelo de Anderson de 1 canal.

\section{CONCLUSÃO}

Neste artigo mostrou-se que o procedimento numérico proposto por Krishna-murthy e colaboradores para a determinação da Temperatura Kondo em modelos de Anderson no regime líquido de Fermi é valido também para modelos de Anderson no regime não líquido de Fermi.

A Temperatura Kondo é um parâmetro importante, pois indica para um dado modelo de Anderson a faixa de temperatura em que passa a ser relevante o acoplamento magnético entre o spin de um átomo e os spins dos elétrons de condução (Efeito Kondo). O procedimento numérico proposto por Krishna-murthy se baseia na análise da curva de suscetibilidade magnética em função da temperatura do átomo acoplado com os elétrons de condução.

Segundo Krishna-murthy e colaboradores, quando a suscetibilidade magnética, multiplicada pela temperatura e por constantes convenientes, apresentar valor igual a uma dada constante $c$, isto é, $k_{B} T \chi /$ $\left(g \mu_{B}\right)^{2} \sim c$, o valor da temperatura é justamente o valor da temperatura Kondo. Krishna-murthy e colaboradores determinaram o valor de $c$ através de um conjunto de dados obtidos por expansão perturbativa. Com estes dados, e utilizando regressão não linear, obteve-se neste artigo o valor $c=0,0698825$.

Para comprovar que o procedimento de Krishna-murthy e colaboradores é correto, mesmo para modelos de Anderson em regime não líquido de Fermi, foi estudado o Modelo de Anderson de Dois Canais. Utilizando os autovalores de energia e os autoestados quânticos obtidos da diagonalização deste modelo através do Grupo de Renormalização Numérica, calculou-se a suscetibilidade magnética e desenharam-se curvas desta grandeza em função da temperatura. Determinou-se a Temperatura Kondo de cada curva usando o procedimento de Krishna-murthy e colaboradores e o valor de $c$ determinado nesse artigo. Estas curvas, devidamente escaladas com os respectivos valores de Temperatura Kondo, resultaram em comportamento universal em baixa temperatura, comprovando que o procedimento é correto. Como era de se esperar, o comportamento universal do Modelo de Anderson em regime não líquido de Fermi é diferente do comportamento universal quando o Modelo de Anderson apresenta regime líquido de Fermi. A técnica de regressão numérica não linear utilizada será de grande ajuda na determinação da dependência da Temperatura Kondo pelos parâmetros físicos do Modelo de Anderson de Dois Canais. 


\section{AGRADECIMENTOS}

Esta pesquisa foi financiada pela FUNDECT-MS.

\section{BIBLIOGRAFIA}

[1] WEYMANN, I., BORDA, L., "Underscreened Kondo effect in quantum dots coupled to ferromagnetic leads", Physical Review B, v. 81, n. 11, pp. 115-445, 2010.

[2] HEWSON, A. C., The Kondo Problem to Heavy Fermions, 1 ed., New York, Cambridge University Press, 1993.

[3] KONDO, J., "Resistance Minimum in Dilute Magnetic Alloys", Progress of Theoretical Physics, v. 32, n. 1 , pp. 37- 49, 1964

[4] ANDERSON, P.W., "Localized Magnetic States in Metals", Physical Review, v. 124, n. 1, pp. 41, 1961.

[5] SCHRIEFFER, J.R., WOLFF, P.A., "Relation between the Anderson and Kondo Hamiltonians", Physical Review, v. 149, n. 2, pp. 491, 1966.

[6] WILSON, K.G., "The renormalization group: Critical phenomena and the Kondo problem", Reviews of Modern Physics, v. 47, n. 4, pp. 773, 1975.

[7] KRISHNA-MUTHY, H.R., WILKINS, J.W, WILSON, K.G., "Renormalization-group approach to the Anderson model of dilute magnetic alloys. I. Static properties for the symmetric case", Physical Review B, v. 21, n. 3, pp. 1003-1043, Feb. 1980.

[8] ANDREI, N., "Diagonalization of the Kondo Hamiltonian", Physical Review Letters, v. 45, n. 5, pp. 379382, 1980.

[9] WIEGMANN, P.B., "Exact solution of the s-d exchange model (Kondo Problem)", Journal Phys. C: Solid State Phys., v. 14, n. 10, pp. 1463-1478, 1981.

[10] ASHCROFT, N.W., MERMIN, N.D., Física do Estado Sólido, São Paulo: Cengage Learning, 2011.

[11] STEWART, G.R., "Non-Fermi liquid behavior in d- and f-electron metals", Reviews of Modern Phisics, v. 73 , n. 4, p. 797-855, Oct. 2001.

[12] Marcone Jamilson Freitas Souza, NOTAS DE AULA DE MÉTODOS NUMÉRICOS, http://www.decom.ufop.br/prof/marcone/Disciplinas/MetodosNumericoseEstatisticos/MetodosNumericos.ht $\underline{m}$, acessado em outubro de 2012 .

[13] SILVA, W.P., SILVA, C.M.D.P.S., SILVA, H.J.G., et al., "Geração de Incertezas de Funções Redutíveis ao Primeiro Grau Ajustada pelo Método dos Mínimos Quadrados", Revista Brasileira de Ensino de Física, v. 21, n. 3, pp. 341, 1999.

[14] SILVA, W.P, SILVA, C.M.D.P.S., CAVALCANTI, C.G.B, et al., "LAB Fit Ajuste de Curvas: Um software em português para tratamento de dados experimentais", Revista Brasileira de Ensino de Física, v. 26, n. 4, pp. 419-427, 2004.

[15] FERREIRA, J.V.B., OLIVEIRA, L.N., COX, D.L., et al., "Non-Fermi liquid fixed points of a twochannel Anderson model", Journal of Magnetism and Magnetic Materials, v. 226-230, n. 1, pp. 196-198, 2001.

[16] NOZIRES, P., BLANDIN, A., "Kondo effect in real metals", Journal de Physique, v. 41, n. 3, pp. 193, 1980.

[17] COX, D.L., MAPLE, M.B., "Electronic Pairing in Exotic Superconductors", Physics Today, v. 48, n. 2, pp. 32, Feb. 1995.

[18] FERREIRA, J.V.B., OLIVEIRA, L.N., COX, D.L., et al., "Magnetic susceptibility of a two-channel Anderson model", Journal of Magnetism and Magnetic Materials, v. 226-230, n. 1, pp. 132-133, 2001.

[19] FERREIRA, J.V.B, LÍBERO, V.L, OLIVEIRA, L.N, "Multi-step transformation in numerical renormalization group", Computer Physics Communications, v. 174, n. 11, pp. 862-868, 2006.

[20] FERREIRA, J.V.B., FERREIRA, A.I.I., LEITE, A.H., et al, "Numerical Renormalization Group computation of temperature dependent specific heat for a two-channel Anderson model", Journal of Magnetism and Magnetic Materials, v. 324, n. 6, pp. 1011-1016, Mar. 2012. 